\title{
DEVELOPMENT OF ELLIPTICAL UNDULATORS FOR ELETTRA
}

\author{
B. DIVIACCO, R. BRACCO, D. MILLO, R.P. WALKER, M. ZALATEU, D. ZANGRANDO \\ Sincrotrone Trieste, 34012 Trieste, Italy
}

\section{INTRODUCTION}

A range of elliptical undulators (EU) has recently been constructed at ELETTRA, whose main parameters are listed in Table 1 below. They are all based on the APPLE-II scheme [1], but two different terminations have been used due to specific installation requirements. Also, one of them (EU12.5) features a quasi-periodic field to suit special beamline needs. The minimum magnetic gap corresponds to the design value of $19 \mathrm{~mm}$ for all the devices, with the exception of one case where it had to be reduced to $18.6 \mathrm{~mm}$ to reach the required field level. It should be pointed out that data for undulator EU4.8 refer to a 10-period prototype, as the full-length device is presently undergoing final assembly.

Table 1: Measured field strength $(\mathrm{T})$ and corresponding fundamental photon energy $(\mathrm{eV})$ for the new undulators

\begin{tabular}{|c|c|c|c|c|c|c|c|}
\hline \multirow[t]{2}{*}{$\begin{array}{l}\text { period } \\
(\mathrm{cm})\end{array}$} & \multirow[t]{2}{*}{$\mathrm{N}$} & \multicolumn{2}{|c|}{$\begin{array}{l}\text { Horizontal } \\
\text { Polarization }\end{array}$} & \multicolumn{2}{|c|}{$\begin{array}{l}\text { Circular } \\
\text { Polarization }\end{array}$} & \multicolumn{2}{|c|}{$\begin{array}{l}\text { Vertical } \\
\text { Polarization }\end{array}$} \\
\hline & & $\mathrm{B}_{0}$ & $\varepsilon_{1}$ & $\mathrm{~B}_{0}$ & $\varepsilon_{1}$ & $\mathrm{~B}_{0}$ & $\varepsilon_{1}$ \\
\hline 4.8 & 44 & 0.58 & 178 & 0.29 & 287 & 0.34 & 366 \\
\hline 6.0 & 36 & 0.78 & 59 & 0.42 & 94 & 0.51 & 123 \\
\hline 7.7 & 28 & 0.92 & 21 & 0.53 & 32 & 0.64 & 43 \\
\hline 10.0 & 20 & 1.02 & 8 & 0.63 & 11 & 0.77 & 14 \\
\hline 12.5 & 17 & 0.77 & 8 & 0.48 & 10 & 0.59 & 13 \\
\hline
\end{tabular}

\section{FIELD QUALITY OPTIMIZATION}

At an early design phase [2] a decision was made that, due to the large number of permament magnet blocks involved, performance optimization should be based on manufacturer's magnetic measurement data. For this reason, an effort was made to specify the allowed magnetic errors on individual blocks in such a way that the material inhomogeneity would be limited as much as technically possible. This was achieved by putting a strict tolerance on the maximum relative difference between the magnetic field measured at some distance (approx. half the minimum gap, or $10 \mathrm{~mm}$ ) above and below two opposite block faces. By limiting this North-South error, and by sorting the blocks in each device based on volumeaveraged magnetization data, we were able to obtain low enough field error levels that a simple post-assembly shimming procedure proved adequate to achieve the required tolerances. This mainly applies to field integral errors, since optical phase errors were always so small (typically less than $3.5^{\circ} \mathrm{rms}$ at any gap and polarization state) that no phase shimming was used except for the very first undulator [3]. The correction method we adopted is based on the application of small horizontal and/or vertical displacements to selected vertically magnetized blocks, a method which has also been used for the ALS elliptical undulator [4] and suggestively named 'virtual shimming'. Figure 1 shows measured quantities for one undulator segment (EU10.0) before and after correction. Note that trajectory straightening is achieved together with significant reduction of first and second order integrated multipoles, while the phase error is not significantly affected. Similar results have been obtained for the other devices, by displacing a maximum of 15 blocks in the worst case.
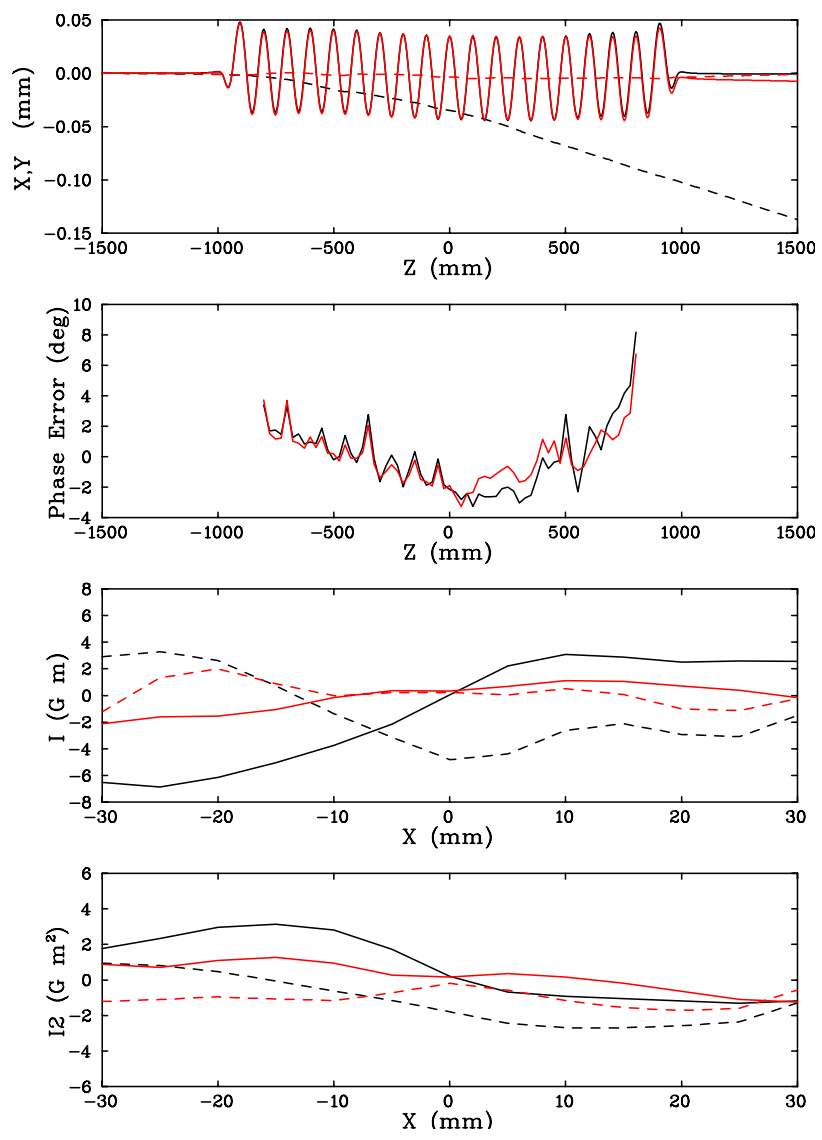

Figure 1: Trajectory, phase error, first and second field integrals for EU10.0 (linear polarization mode) before (black) and after (red) shimming. Continuous line: vertical field, dotted lines: horizontal field component.

\section{TERMINATIONS}

End terminations for the EU6.0, EU12.5 and EU10.0 undulators were designed with the primary objective of reducing as much as possible the fringe-field level. This was motivated by the close distance between these devices 
and two additional electromagnets which have been installed in the center of the straight sections, in one case (EU10.0) to realize the 'optical klystron' needed for FEL operation, in the other (EU6.0+EU12.5) to implement an electron beam chicane providing angular separation of the two radiation sources. This was achieved with a (displacement free) termination schematically shown in figure 2a. The drawback of this design is a significant variation of field integrals with the longitudinal phasing of the arrays $[2,3,4,5]$. This effect, determined by the nonunit permeability of the magnetic material used $(\mathrm{NdFeB}$ in the present case), can be minimized by a different end scheme [5]. By relaxing the requirements on fringe-field and trajectory displacement, a different termination was designed for the other two undulators (EU4.8, EU7.7). For the sake of simplicity, it makes use only of already available full and half-size blocks (see figure $2 \mathrm{~b}$ ) with some (numerically optimized) spaces $(\mathrm{s} 1, \mathrm{~s} 2, \mathrm{~s} 3)$ between the first three pair of blocks.

a

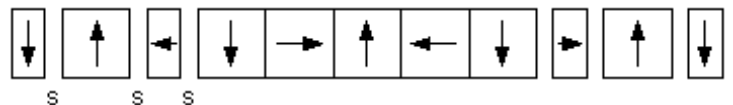

b

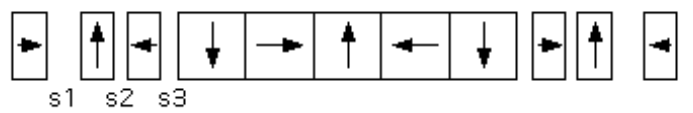

Figure 2: Low fringe-field termination $\left(s=\lambda_{0} / 16\right)$ as used for EU6.0, EU10.0, EU12.5 and low field integral termination as used for EU4.8 $(\mathrm{s} 1=5 \mathrm{~mm}, \mathrm{~s} 2=\mathrm{s} 3=0)$ and EU7.7 (s1=6mm, s2=s3=0).

Figure 3 shows the difference in fringe field between two undulators (EU10.0 and EU7.7) having similar peak field amplitudes but different terminations.

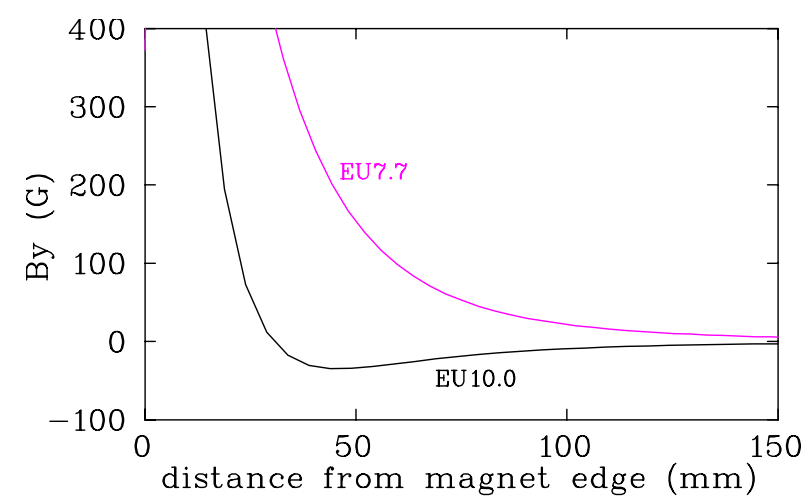

Figure 3: Measured fringe field distributions for EU10.0 (type-a termination) and EU7.7 (type-b termination).

For symmetric terminations like these, the ( $\mu-1)$ effect shows as a variation of the vertical first field integral (Iy) and the horizontal second field integral (I2x) with the phasing of the arrays (Zs), as shown in figure 4 and 5 . The much smaller variation of these integrals with phase for the second termination (EU4.8, EU7.7) compared to the first (EU10.0, EU12.5) is very evident. It should be noted that for an anti-symmetric termination, such as that used in [4] the effect is reversed, generating a variation of the horizontal first field integral (Ix) and the vertical second field integral (I2y).

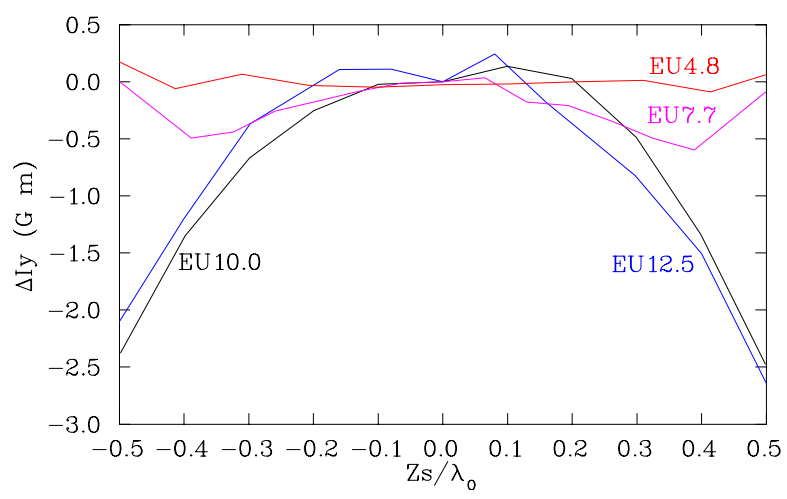

Figure 4: First vertical field integral as a function of array phasing for different undulators.

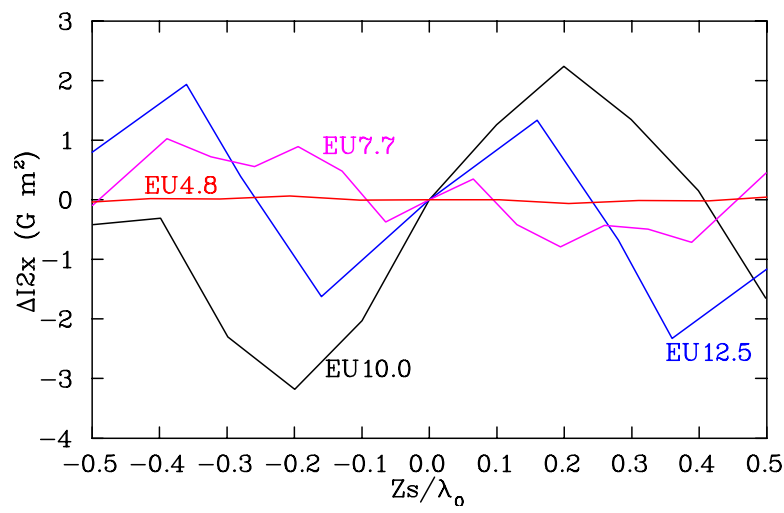

Figure 5: As fig. 4 for the second horizontal field integral.

\section{QUASI PERIODIC UNDULATOR}

Quasi-periodic undulators have been originally proposed [6] as a method to reduce contamination from high order spectral harmonics whenever optical filtering is not possible or convenient. In the first implementation, quasiperiodicity was obtained by changing the length of magnetic field half-periods according to a suitable sequence of long and short spaces. We have developed an alternative solution in which the field amplitude (rather than the period) is modulated along the length of the device $[2,7]$. This is achieved by selectively removing a few horizontally magnetized blocks, which can be done without any modification to the standard magnet holders. This concept has been applied to the EU12.5 variable polarization undulator. Figure 6 shows the measured field distribution in the circular polarization mode, clearly showing the four locations of perturbed field due to the missing blocks. The corresponding computed trajectory is oscillating piece-wise along two different axes in a very similar fashion as in the standard variable-spacing quasiperiodic device. The computed spectrum is shown in figure 7 for a realistic case where the relatively large 
beamline angular acceptance is included. It can be seen that good harmonic suppression is achieved for linearly polarized radiation with no performance penalty in the circular polarization mode.
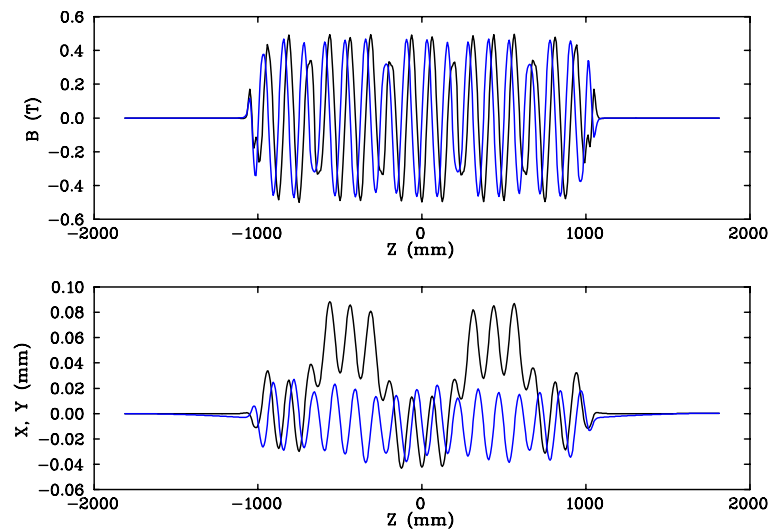

Figure 6: Measured field and corresponding trajectory for the quasi-periodic undulator in circular polarization mode. Vertical field (horizontal trajectory) shown in black, horizontal field (vertical trajectory) in blue.
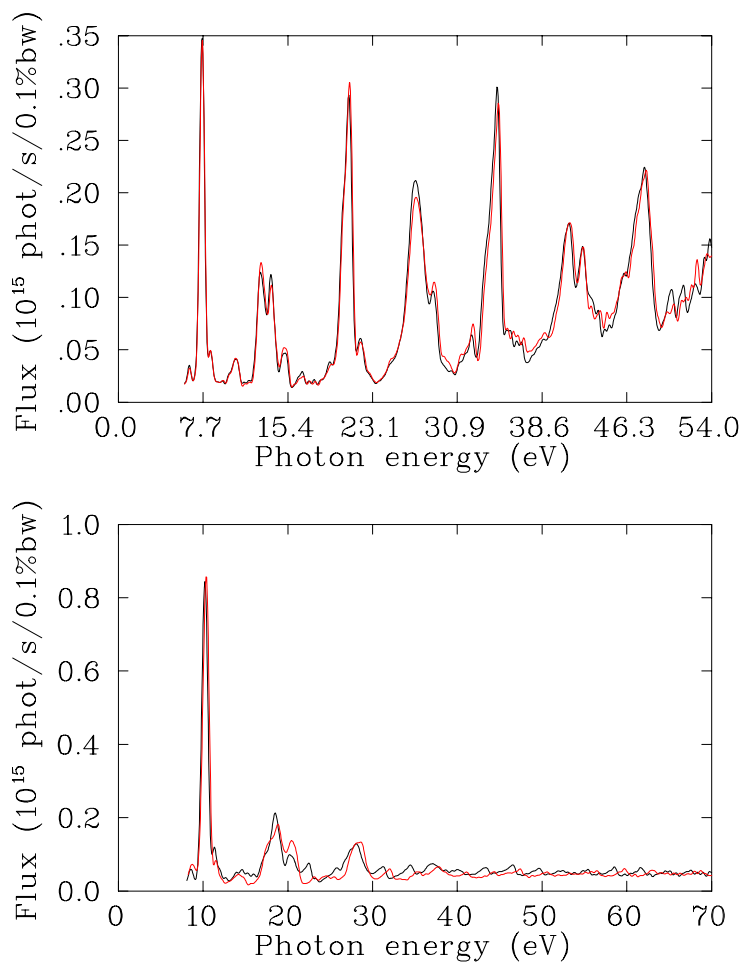

Figure 7: Angle-integrated spectra of EU12.5 in linear (upper) and circular (lower) polarization modes, computed from the ideal (black line) and measured fields (red line). Angular acceptance is 0.7 x $0.7 \mathrm{mrad}$.

\section{FOCUSING PROPERTIES}

Undulator focusing is determined by the field variation as experienced by a particle travelling along the device offaxis. In the standard vertical field device made of two rows of magnet arrays, focusing strength is positive in the vertical plane and negative (= defocusing) in the horizontal plane, gradually decreasing as the field is reduced by opening the magnetic gap. The situation is more complicated for adjustable polarization devices due to the varying ratio between horizontal and vertical field amplitudes and to the different transverse off-axis field distributions created by the four rows of magnets. In general, a numerical calculation is needed to accurately predict the way the focusing strength changes with gap and array shifting [8]. Table 2 shows the results of this calculation based on the ideal 3D field of the different undulators.

Table 2: Horizontal / vertical second-order focusing strength $\left(\mathrm{K}_{\mathrm{X}} \mathrm{L} / \mathrm{K}_{\mathrm{Y}} \mathrm{L}\right)$ in $\mathrm{T}^{2} \cdot \mathrm{m}$ for the new undulators

\begin{tabular}{llll}
\hline device & $\begin{array}{l}\text { Horizontal } \\
\text { Polarization }\end{array}$ & $\begin{array}{l}\text { Circular } \\
\text { Polarization }\end{array}$ & $\begin{array}{l}\text { Vertical } \\
\text { Polarization }\end{array}$ \\
\hline EU4.8 & $0.02 / 0.69$ & $-0.21 / 0.57$ & $-0.28 / 0.53$ \\
EU6.0 & $0.12 / 1.17$ & $-0.44 / 1.19$ & $-0.71 / 1.28$ \\
EU7.7 & $0.21 / 1.55$ & $-1.00 / 2.16$ & $-1.57 / 2.45$ \\
EU10.0 & $0.34 / 1.69$ & $-1.97 / 3.47$ & $-3.31 / 4.53$ \\
EU12.5 & $0.12 / 1.07$ & $-2.31 / 3.22$ & $-3.60 / 4.37$ \\
\hline
\end{tabular}

Notice that, in the horizontal polarization (vertical field) mode, the devices are focusing in both planes $\left(\mathrm{K}_{\mathrm{X}, \mathrm{Y}} \mathrm{L}>0\right)$, unlike conventional flat-pole planar undulators which are horizontally defocusing. Focusing strengths rapidly increase when changing to elliptical / circular / vertical polarization. The effect is stronger for higher field devices and, for the same field, for longer periods. The associated e-beam tune shift is given by:

$$
\Delta Q_{x, y}=\frac{1}{2}\left(\frac{e}{\gamma m c}\right)^{2} \frac{K_{x, y} L \cdot \beta_{x, y}}{4 \pi}
$$

This has been measured for the already operational undulators (EU6.0, EU12.5, EU10.0), and found in good agreement with the above calculations [9].

\section{REFERENCES}

[1] S. Sasaki, Nucl. Instr. and Meth. in Phys. Res. A 347 (1994) pag. 83.

[2] B. Diviacco et al., proc. 1998 European Particle Accelerator Conference, pag. 2216.

[3] B. Diviacco et al., proc. 1999 Particle Accelerator Conference, pag. 2680.

[4] S. Marks et al., proc. 1999 Particle Accelerator Conference, pag. 162.

[5] J. Chavanne et al., proc. 1999 Particle Accelerator Conference, pag. 2665.

[6] S. Hashimoto and S. Sasaki, Nucl. Instr. and Meth. in Phys. res. A361 (1995) pag. 611.

[7] B. Diviacco and R.P.Walker, Sincrotrone Trieste Technical Note ST/M-97-11, March 1997.

[8] P. Elleaume, Proc. 1992 European Particle Accelerator Conference, pag. 661.

[9] L. Tosi et al., these procedeengs. 